\title{
Single Molecule Lifetime Fluctuations Reveal Segmental Dynamics in Polymers
}

\author{
R. A. L. Vallée, ${ }^{1,2}$ N. Tomczak, ${ }^{2}$ L. Kuipers, ${ }^{1}$ G. J. Vancso, ${ }^{2}$ and N. F. van Hulst ${ }^{1, *}$ \\ ${ }^{1}$ Applied Optics Group, MESA ${ }^{+}$Research Institute, University of Twente, P.O. Box 217, 7500 AE Enschede, The Netherlands \\ ${ }^{2}$ Materials Science and Technology of Polymers, MESA ${ }^{+}$Research Institute, University of Twente, \\ P.O. Box 217, 7500 AE Enschede, The Netherlands
}

(Received 6 March 2003; published 17 July 2003)

\begin{abstract}
We present a single molecule fluorescence study that allows one to probe the nanoscale segmental dynamics in amorphous polymer matrices. By recording single molecular lifetime trajectories of embedded fluorophores, peculiar excursions towards longer lifetimes are observed. The asymmetric response is shown to reflect variations in the photonic mode density as a result of the local density fluctuations of the surrounding polymer. We determine the number of polymer segments involved in a local segmental rearrangement volume around the probe. A common decrease of the number of segments with temperature is found for both investigated polymers, poly(styrene) and poly(isobutylmethacrylate). Our novel approach will prove powerful for the understanding of the nanoscale rearrangements in functional polymers.
\end{abstract}

DOI: 10.1103/PhysRevLett.91.038301

Glasses are disordered materials usually obtained by cooling a viscous liquid or a polymer melt fast enough to avoid crystallization. Their static and dynamic properties deviate largely from the simple Debye behavior. The deviations are best interpreted in terms of dynamic heterogeneities of structure on the segmental (nanometer) scale [1]. Direct evidence for microscopic regions of different relaxation time has been obtained by multidimensional nuclear magnetic resonance [2], photobleaching [3], excess light scattering near the glass transition temperature $\left(T_{g}\right)$ [4], dielectric hole burning [5], and, recently, single molecule spectroscopy [6]. However, the key question concerning the temperature dependence of the characteristic dimensions of the inhomogeneities in glass forming liquids and amorphous solids is still unanswered.

Single molecule detection has proven to be a unique method to investigate the behavior of complex condensed systems $[7,8]$. In contrast to ensemble methods, the single molecule approach provides information on time trajectories, distributions, and correlations of observables that would otherwise be hidden. Individual members of a heterogeneous population are examined, identified, and sorted to quantitatively compare their subpopulations. In the extreme case of cryogenic temperatures, it has been shown [9] that most of the spectral trails of single molecules obtained at around $1 \mathrm{~K}$ are consistent with the standard two-level system model of glasses [10]. At room temperature, the broad spectra and complexity of the system complicate the single molecule spectral analysis.

We present in this Letter a first study in the complementary time domain. The excited state lifetime of the individual dye molecules is monitored in time. In a static environment, the lifetime has a discrete value. Because of the heterogeneity of the nanoenvironment, the lifetime is different but constant for every molecule $[11,12]$. However, in a fluctuating environment, the lifetime will
PACS numbers: 82.37.-j, 05.40.-a, 33.50.-j, 82.35.Np

vary and develop a certain distribution. We show that lifetime fluctuations are due to variations of the radiative density of states (RDOS) and consequently reflect the local density fluctuations in the surroundings of the single molecule probe. We establish in a direct way the number of segments involved in the rearrangement volume surrounding the fluorophore, by connecting our observations to the Simha-Somcynsky (SS) equation of state [13]. Interestingly, a common decrease of the number of segments with temperature for different polymers is found, in agreement with the predictions of the thermodynamic Adam-Gibbs theory [14].

Dye-doped polymer films (70 and $200 \mathrm{~nm}$ ) were prepared by spin coating a solution of 1,1'-dioctadecyl3, 3, 3, 3' - tetramethylindodicarbocyanine (DiD, 5 × $10^{-10} \mathrm{M}$, Molecular Probes) and polystyrene [PS, $89300 \mathrm{~g} / \mathrm{mol}$, polydispersity index (PI) of 1.06, Polymer Standard Service], or poly(isobutylmethacrylate) (PIBMA, $67200 \mathrm{~g} / \mathrm{mol}, \mathrm{PI}=2.8$, custom made radical polymerization) in toluene onto a glass substrate. Further annealing was performed in order to relax the stresses induced by the deposition procedure. The choice of the dye was dictated by the following considerations: it possesses a high fluorescence quantum yield (close to unity), an absorption cross section of $7.5 \times 10^{-16} \mathrm{~cm}^{2}$, and is highly photostable when embedded in a polymer matrix [11]. PS $\left(T_{g}=100^{\circ} \mathrm{C}\right)$ and PIBMA $\left(T_{g}=56^{\circ} \mathrm{C}\right)$ were chosen due to to their different glass transition temperatures $T_{g}$. This allows us to probe polymer properties as a function of their relative distance to $T_{g}$, while working at the same laboratory temperatures for both polymers. Molecules in the sample were excited by $57 \mathrm{ps}$ pulses at a wavelength of $635 \mathrm{~nm}$ and repetition rate of $80 \mathrm{MHz}$, generated by a ps pulsed diode laser (PicoQuant, PDL 800-B, $100 \mu \mathrm{W}$ ), at the focus of a confocal inverted microscope (Zeiss). Fluorescence intensity and lifetime of individual molecules were monitored in time, in 
consecutive experiments, using a time-correlated singlephoton counting card (Becker \& Hickl, SPC 500) [15]. Integrating over $200 \mathrm{~ms}$ time intervals, a lifetime accuracy of typically $0.1-0.3 \mathrm{~ns}$ was obtained.

Figure 1 shows fluorescence lifetime trajectories of two individual molecules emitting at approximately the same intensity level. Both trajectories fluctuate in time, but in a different way: while the first molecule (a) has a lifetime restricted to small and rather symmetrical variations around the mean value, the second molecule dwells occasionally longer in the excited state, as is clear from the excursions to longer lifetimes in the transient (b). The corresponding single molecule fluorescence lifetime distributions built up from the transients further underline the difference in behavior between the two molecules. The characteristic shape varies from nearly symmetric (c) to asymmetric (d). Why does the fluorescence lifetime change? Three factors may potentially affect the fluorescence lifetime of dyes embedded in a dielectric medium: quenching effects, changes in the conformation of the fluorophore, and variations in the dielectric properties surrounding the probe molecule.

First, quenching effects may be discarded since the aperture of nonradiative decay channels in the matrix would simultaneously induce a lowering of the measured intensity and lifetime. This is not the case since intensity and lifetime are not correlated in the plots of Fig. 1. Furthermore, the quantum efficiency of the chosen fluorophore (close to unity) excludes the possibility that the large lifetime fluctuations result from fluctuations in the number of decay channels. Moreover, the excursions in the fluorescence lifetime trajectory [Fig. 1(b)] are always towards higher values, which rules out any nonradiative process as the cause of the fluctuations.

Second, the electronic properties of the conjugated DiD molecule are dominated by the presence of delocalized $\pi$ electrons. The lowest optical excitation corresponds to a $\pi \rightarrow \pi *$ transition and vice versa for the emission. The molecule may thus be described as a simple two-level system. In the electric dipole approximation and in vacuum, the spontaneous emission rate $\Gamma_{0}$ of the molecule is given by the relation [16]

$$
\Gamma_{0}=\frac{\omega_{0}^{3} d^{2}}{3 \pi \epsilon_{0} \bar{h} c^{3}} .
$$

The radiative lifetime $\tau_{0}$ is the inverse of $\Gamma_{0}$, where $\omega_{0}, d$, and $\epsilon_{0}$ designate the transition frequency, the transition dipole moment of the excited state of the fluorophore for the $\pi \rightarrow \pi *$ transition, and the dielectric constant of the vacuum, respectively. This equation reveals that a change in the transition frequency or the transition dipole moment of the dye due to the influence of the nanoenvironment can be responsible for the observed fluctuations of the lifetime. However, by quantum chemistry calculations, we showed [17] that low-cost energy motions of the DiD molecule compatible with thermal agitation at room temperature result in a fluctuation of at most $10 \%$ in the fluorescence lifetime when compared to the rest structure. Therefore conformational changes do not explain the large fluctuations observed.
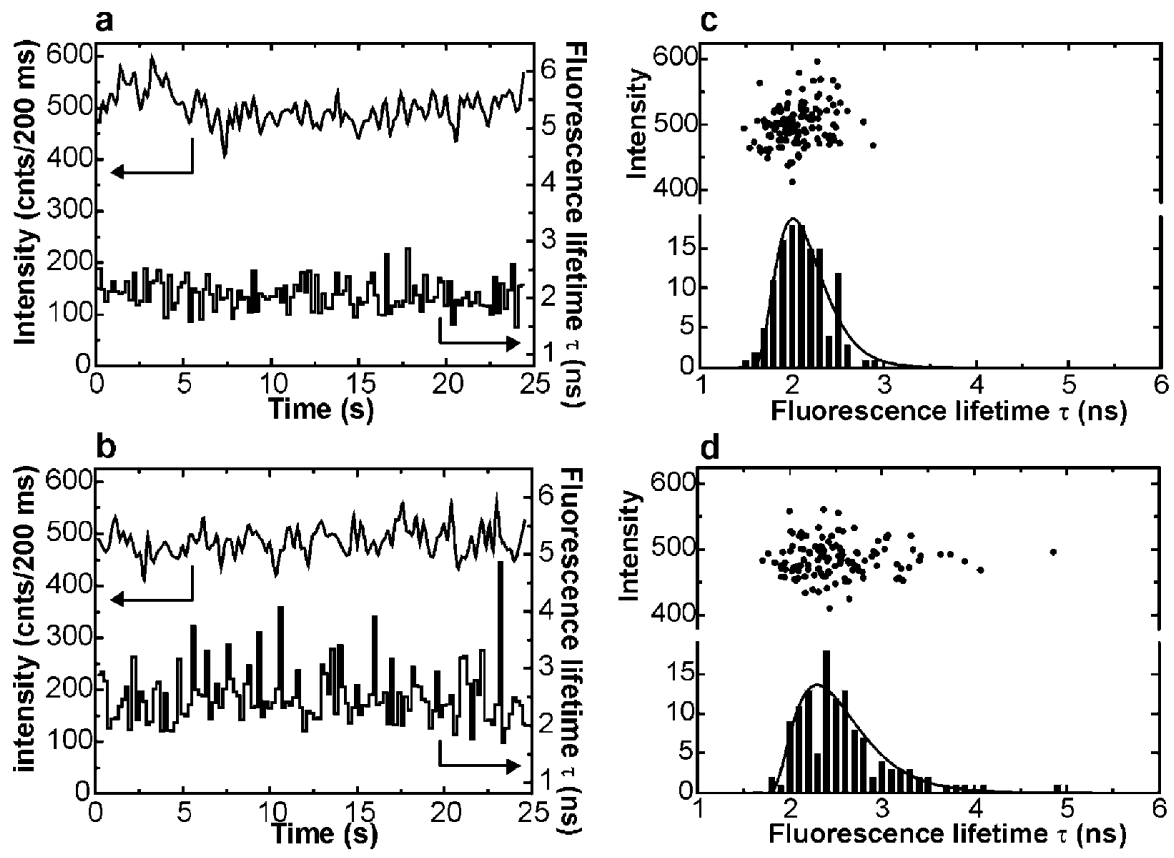

FIG. 1. (a),(b) Transients of fluorescence intensity and fluorescence lifetime for two different individual DiD molecules embedded in a $200 \mathrm{~nm}$ thick film of PIBMA at room temperature. Clearly, the second molecule exhibits excursions towards longer lifetime values. (c),(d) For the two transients corresponding fluorescence lifetime distributions (bottom) and correlation between intensity and lifetime (top) are shown. The shape of the distribution is more asymmetric for the second molecule. While the intensity remains within a $10 \%$ fluctuating range, the lifetime shows changes up to $100 \%$. 


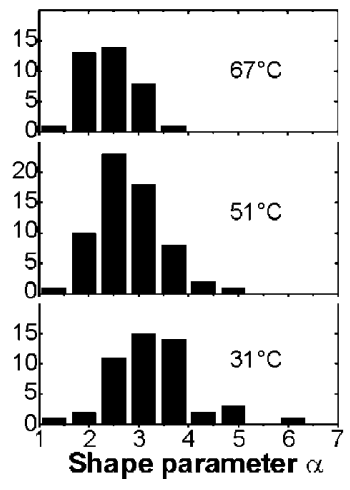

FIG. 2. Distributions of shape parameter for DiD molecules embedded in a $200 \mathrm{~nm}$ thick film of PIBMA for three temperatures. With increasing temperature, the distributions shift towards lower shape values.

As a result, we attribute the radiative lifetime fluctuations observed to the local density fluctuations of the surrounding polymer matrix. We quantified this effect for 778 individual probe molecules embedded in different polymer matrices, at various temperatures. For each single molecule trajectory lasting at least $15 \mathrm{~s}$, we were able to build up a reliable distribution of fluorescence lifetimes. Interestingly, the experimental lifetime distribution is best fitted with a gamma distribution $g(x)$. This distribution $g(x)=\beta(\beta x)^{\alpha-1} e^{-\beta x} / \Gamma(\alpha)$ is generally accepted for a lower bounded continuous variable fully characterized by its first two moments $x_{\mathrm{av}}$ and $\left\langle\delta x^{2}\right\rangle_{\mathrm{av}}$ or, equivalently, by its shape $(\alpha)$ and scale $(\beta)$ parameters, where $x_{\mathrm{av}}=\frac{\alpha}{\beta},\left\langle\delta x^{2}\right\rangle_{\mathrm{av}}=\frac{\alpha}{\beta^{2}}$, and $\Gamma(\alpha)$ stands for the gamma function. From the fitted gamma distributions, we extract the value of the characteristic shape parameter $\alpha$. At each given temperature, collecting the shape parameters of 30 to 60 of these molecules, we constructed the distribution of the shape parameter. Figure 2 shows the shape distributions for three different temperatures for DiD embedded in a $200 \mathrm{~nm}$ thick film of PIBMA. Surprisingly, upon increasing temperature, the average shape value as well as the width of the shape parameter distribution decrease. This temperature dependence further corroborates the conclusion that the density fluctuation in the polymer is the main factor in the observed lifetime fluctuations.

How can density changes within the polymer affect the fluorescence lifetime of an embedded fluorophore? The probe molecule is placed in a nonoccupied space (cavity) of a polymeric matrix. The energy it radiates thus depends on the dielectric properties of the local surroundings. The spontaneous emission rate $\Gamma(\epsilon)$ of the dye inside a homogeneous medium with dielectric constant $\epsilon$ is predicted to follow the $\sqrt{\epsilon}$ dependence of the RDOS [18]: $\Gamma(\epsilon)=$ $\sqrt{\epsilon} \Gamma_{0}$. However, this result was obtained by quantizing the macroscopic electromagnetic field, while the dipole couples to the local field at the position of the molecule. Strictly the dipole-dipole interaction considering the surrounding polymeric voids should be calculated [19]; in

038301-3 practice, for complex systems, the concept of local field is introduced. The microscopic local field differs from the macroscopic field by a local field correction factor $L_{f}$ given by

$$
L_{f}=\frac{3 \epsilon}{2 \epsilon+1-\frac{2 \alpha}{3 \Omega}(\epsilon-1)},
$$

where here $\alpha$ is the polarizability of the dye in a cavity of volume $\Omega$ [20]. For substitutional or interstitial impurities such as dyes in an otherwise homogeneous medium, Eq. (2) reduces to the empty-cavity (Lorentz) local field factor $L_{f}=\frac{3 \epsilon}{2 \epsilon+1}$. For dye molecules with a substantial polarizability the local field fluctuations might exceed the Lorentz factor. By including the local field factor the spontaneous emission rate is given by $\Gamma(\epsilon)=L_{f}^{2} \sqrt{\epsilon} \Gamma_{0}$.

Up to now, the polymer matrix has been considered as a homogeneous medium with a dielectric constant $\epsilon$. However, polymer chain segments move in time in the polymer matrix at room temperature. For the probe molecule, the segmental rearrangements imply either creation or annihilation of voids in its nanoenvironment, and thus a change of its surrounding local dielectric constant. Consequently, the system has to be considered as an effective medium [21], consisting of polymer segments and voids competing to occupy space. A local effective dielectric constant $\epsilon$ modulated by the fraction $h$ of holes present in the medium is given by

$$
\epsilon=h \epsilon_{\mathrm{vac}}+(1-h) \epsilon_{\mathrm{pol}},
$$

where $\epsilon_{\mathrm{vac}}=1$ and $\epsilon_{\mathrm{pol}}=2.5$ designate the vacuum and polymer (PS) dielectric constant, respectively.

Following usual statistical theory, the change of variables $\tau$ into $h$ is accompanied by a corresponding change of the probability density $f(\tau)$ into $g(h)=f(\tau)\left|\frac{d \tau}{d h}\right|$. For the effective medium considered here, this dependence is smoothly linear: converting a gamma distribution of holes, characterized by its first two moments $h_{\mathrm{av}}=10 \%$ and $\left\langle\delta x^{2}\right\rangle_{\mathrm{av}}=0.4 \%$ to the corresponding distribution of lifetimes, leads to a nonlinear deviation of only $5 \%$ in the corresponding value of the shape parameter.

To relate our claim to a classical polymer theory, it is interesting to note that the SS model [13] considers the polymer as a lattice of sites that can accommodate the chain segments of macromolecules. To account for molecular disorder, a temperature and volume dependent fraction $h$ of holes is introduced. Knowing the configurational properties of the system, an equation of state has been established [22], which permits the determination of the fractional mean free volume $h_{\mathrm{av}}$ present in the system. However, due to thermal fluctuations, the free volume varies both in time and at every position. The mean-squared deviation from the mean free volume can be calculated once the number of polymer segments $\left(N_{s}\right)$ involved in a segmental rearrangement cell is known. From the first two moments and by attributing a value to $N_{s}$, a gamma distribution of free volume is built $[23,24]$. Given a gamma distribution of free volume, the 


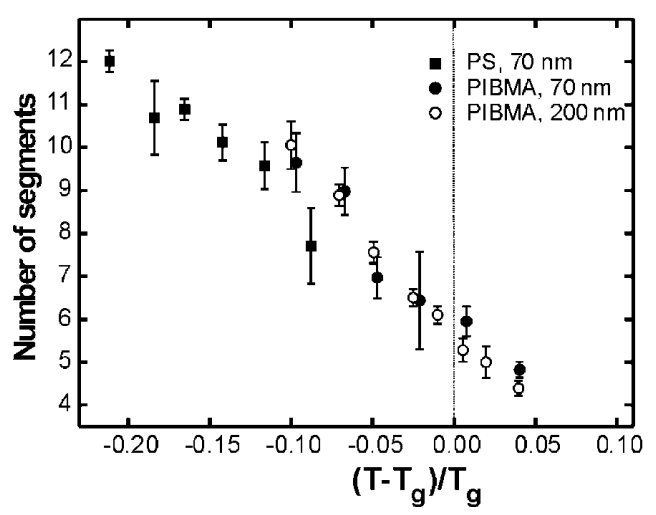

FIG. 3. Master plot of the number of segments $N_{s}$ involved in a segmental rearrangement cell as a function of the reduced temperature $\left(T-T_{g}\right) / T_{g}$ for PS (squares, $T_{g}=100{ }^{\circ} \mathrm{C}$ ) and for PIBMA (circles, $T_{g}=56^{\circ} \mathrm{C}$ ). In the case of PIBMA, data for both a $70 \mathrm{~nm}$ thick film (closed circles) and a $200 \mathrm{~nm}$ thick film (open circles) are shown. At the glass transition temperature, typically six segments play a role in the segmental rearrangement.

number of segments $\left(N_{s}\right)$ becomes a linear function of the shape parameter $\alpha$ multiplied by a temperature and volume dependent factor, $N_{s}=\alpha f(h, \tilde{V}, \tilde{T})$. The shape distributions, as shown in Fig. 2, can therefore be converted into corresponding distributions of the number of segments $N_{s}$ involved in a local segmental rearrangement cell.

Figure 3 shows the peak positions of the $N_{s}$ distributions as a function of temperature for PIBMA, 70 and $200 \mathrm{~nm}$ thick films, and PS, $70 \mathrm{~nm}$ thick film. The striking feature of this master plot is the appearance of a general behavior, with the reduced temperature $\left(T-T_{g}\right) / T_{g}$ as a common parameter. The observation of the decrease of the number of segments when increasing temperature is in agreement with the configurational entropy model of Adam and Gibbs (AG), which predicts that the length scale of the cooperatively rearranging regions (CRR) decreases with increasing temperature. Furthermore, the changes in width and position of the distributions with temperature, shown in Fig. 2, clearly reveal the existence of microheterogeneous domains of different sizes and relaxation times, which is not considered in the AG theory, where the CRRs are assumed to be equivalent. Admitting a distribution of sizes of the independently relaxing CRRs, the theory thus does not give any guidance as to what the distribution should be. Single molecule spectroscopy and Fig. 2 provide such distributions and even show a reduction in the width of the distributions with increasing temperature, related to the appearance of a more homogeneous dynamics as the temperature is increased.

Having opened a route towards direct microscopic insight in the segmental dynamics of polymers, it will be interesting to explore the significance of the $N_{s}$ value around $T_{g}$ and the behavior towards higher temperatures.
We believe that our novel approach has large potential for the understanding of the nanoscale dynamics of functional polymers and biopolymers.

The authors are grateful to Jeroen Korterik and Frans Segerink for technical support, Erik van Dijk for TCSPC interfacing and helpful discussion of the results, and María García-Parajó for introducing the first author to the single molecule field. This research is supported by the Council for Chemical Sciences of the Netherlands Organisation for Scientific Research (NWO-CW).

*Electronic address: n.f.vanhulst@tn.utwente.nl

[1] C. A. Angell, K. L. Ngai, G. B. McKenna, P. F. McMillan, and S.W. Martin, J. Appl. Phys. 88, 3113 (2000).

[2] U. Tracht, M. Wilhem, A. Heuer, H. Feng, K. SchmidtRohr, and H.W. Spiess, Phys. Rev. Lett. 81, 2727 (1998).

[3] M. D. Ediger, Annu. Rev. Phys. Chem. 51, 99 (2000).

[4] C.T. Moynihan and J. Schroeder, J. Non-Cryst. Solids 160, 52 (1993).

[5] B. Schiener, A. Loidl, R. Bohmer, and R. V. Chamberlin, Science 274, 752 (1996).

[6] L. A. Deschesnes and D. A. Vanden Bout, Science 292, 255 (2001).

[7] X. S. Xie and J. K. Trautman, Annu. Rev. Phys. Chem. 49, 441 (1998).

[8] W. E. Moerner and M. Orrit, Science 283, 1670 (1999).

[9] A.-M. Boiron, Ph. Tamarat, B. Lounis, and M. Orrit, Chem. Phys. 247, 119 (1999).

[10] E. Geva and J. L. Skinner, J. Chem. Phys. 109, 4920 (1998).

[11] J. J. Macklin, J. K. Trautman, T. D. Harris, and L. E. Brus, Science 272, 255 (1996).

[12] J. A. Veerman, M. F. Garcia Parajo, L. Kuipers, and N. F. van Hulst, Phys. Rev. Lett. 83, 2155 (1999).

[13] R. Simha and T. Somcynski, Macromolecules 2, 342 (1969).

[14] G. Adam and J. H. Gibbs, J. Chem. Phys. 43, 139 (1965).

[15] R. A. L. Vallée, N. Tomczak, H. Gersen, E. M. P. H. van Dijk, M. F. Garcia-Parajo, G. J. Vancso, and N. F. van Hulst, Chem. Phys. Lett. 348, 161 (2001).

[16] R. J. Glauber and M. Lewenstein, Phys. Rev. A 43, 467 (1991).

[17] R. A. L. Vallée, G. J. Vancso, N. F. van Hulst, J.-P. Calbert, J. Cornil, and J. L. Brédas, Chem. Phys. Lett. 372, 282 (2003).

[18] G. Nienhuis and C. Th. J. Alkemade, Physica (Amsterdam) 81C, 181 (1976).

[19] E. A. Donley, H. Bach, U. P. Wild, and T. Plakhotnik, J. Phys. Chem. A 103, 2282 (1999).

[20] F. J. P. Schuurmans, P. de Vries, and A. Lagendijk, Phys. Lett. A 264, 472 (2000).

[21] D. E. Aspnes, Am. J. Phys. 50, 704 (1982).

[22] R. Simha, Macromolecules 10, 1025 (1977).

[23] R. E. Robertson, R. Simha, and J.G. Curro, Macromolecules 17, 911 (1984).

[24] R. E. Robertson, Computational Modeling of Polymers, Free-Volume Theory and Its Application to Polymer Relaxation in the Glassy State, edited by J. Bicerano (Marcel Dekker, Inc., New York, 1992). 